Revue des patrimoines

$42 \mid 2020$

Imagerie numérique et patrimoine culturel :

représentation et transmission des connaissances

\title{
Les enjeux de la narration dans l'immersion 3D archéologique
}

Narration issues in archaeological 3D immersion

Laurent Lescop, Jean-Michel Geneste, Martin Marquet, Victor Yvin, Pacôme Gérard et Pascal Magontier

\section{(2) OpenEdition}

Journals

Édition électronique

URL : http://journals.openedition.org/insitu/27623

DOI : $10.4000 /$ insitu. 27623

ISSN : 1630-7305

Éditeur

Ministère de la Culture

Référence électronique

Laurent Lescop, Jean-Michel Geneste, Martin Marquet, Victor Yvin, Pacôme Gérard et Pascal

Magontier, «Les enjeux de la narration dans l'immersion 3D archéologique », In Situ [En ligne], 42 I

2020, mis en ligne le 12 juin 2020, consulté le 12 janvier 2021. URL : http://journals.openedition.org/ insitu/27623 ; DOI : https://doi.org/10.4000/insitu.27623

Ce document a été généré automatiquement le 12 janvier 2021.

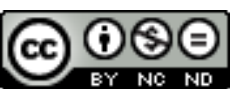

In Situ Revues des patrimoines est mis à disposition selon les termes de la licence Creative Commons Attribution - Pas d'Utilisation Commerciale - Pas de Modification 4.0 International. 


\title{
Les enjeux de la narration dans l'immersion 3D archéologique
}

\author{
Narration issues in archaeological 3D immersion \\ Laurent Lescop, Jean-Michel Geneste, Martin Marquet, Victor Yvin, \\ Pacôme Gérard et Pascal Magontier
}

\section{Introduction}

1 En une vingtaine d'années, les techniques numériques graphiques ont quitté les domaines exclusifs des secteurs académiques et industriels pour irriguer des secteurs de production orientés vers le grand public parmi lesquels notamment les industries créatives, ludiques et culturelles. Ce mouvement ne s'est pas fait sans résistances. Antoine Picon ${ }^{1}$, commentant le domaine de l'architecture, rappelle que la question a fait l'objet « d'une littérature essentiellement doctrinale voire doctrinaire émanant de prosélytes, de technophiles et autres prophètes de microscopiques néo-avant-gardes ». L'arrivée des digital natives, cette génération née avec le $\mathrm{xxI}^{\mathrm{e}}$ siècle, a instauré une nouvelle donne en mettant en phase la disponibilité des outils, la relative facilité de leur apprentissage, la massification de leur diffusion et une meilleure acceptation de la part des générations qui précèdent. La médiation patrimoniale s'inscrit dans ce mouvement: elle s'intègre dans une vision globale de ville ou de territoire palimpseste $^{2}$, une "ville intelligente, connectée, (qui) favorise le développement de nouvelles technologies à travers de nombreuses applications qui permettront de développer notre mobilité et notre autonomie. Nous sommes au début d'une ère d'accélération du changement ${ }^{3} \ldots$ ".

2 Il convient toutefois de ne pas confondre les évolutions techniques portées par des améliorations instrumentales et le renouvellement des paradigmes liés à la représentation et à la communication des connaissances. Si les nouvelles technologies ont épuisé les anciens paradigmes, alors nous devons être particulièrement attentifs à ceux qui se présentent à nous. Si, à l'inverse, il ne s'agit que d'une reformulation, notre 
rôle est de tracer les continuités entre des préoccupations anciennes et leur mise en tension actuelle.

3 La représentation tridimensionnelle, par exemple, a ouvert de nombreuses nouvelles possibilités de figuration et de médiation. Si la figuration ne s'est guère écartée des conventions issues de la perspective monofocale, la médiation s'aventure, avec le numérique, sur les chemins de l'immersion, vers la possibilité de projeter un spectateur dans un ailleurs spatial ou temporel, existant, restitué ou rêvé. Nous ne sommes toutefois pas dupes des biais culturels que les systèmes de représentation embarquent. Portant l'illusion de retranscrire une vision naturelle, la plupart des outils numériques que nous utilisons pour créer des espaces tridimensionnels utilisent les lois de la perspective classique dite "de Brunelleschi». Or les tentatives de transcription virtuelles de la peinture chinoise par exemple ${ }^{4}$ et la grande difficulté à s'affranchir des déterminants culturels inscrits en dur dans les logiciels. Qu'en est-il lorsque nous évoquons la préhistoire? De qui les restitutions 3D constituent-elles le point de vue? Du contemporain des artéfacts ou de celui du chercheur? Si bien que l'immersion, en tant que médiation en archéologie, pourrait être perçue comme un tour de manège sophistiqué, alors qu'on attend d'elle qu'elle ouvre le chercheur et le public à l'appréhension phénoménologique des sites présentés, qu'elle produise une mise en contexte permettant d'approcher, à défaut de saisir, les interactions complexes entre une production anthropique, un espace parfois aménagé et des percepts. Le placement des figures de lions sur les parois de la grotte Chauvet-Pont d'Arc (Vallon-Pont d'Arc, Ardèche, France), par exemple, ne peut se comprendre que par des jeux d'alignement que l'immersion est parfaitement capable de faire percevoir.

\section{Références de dispositifs immersifs}

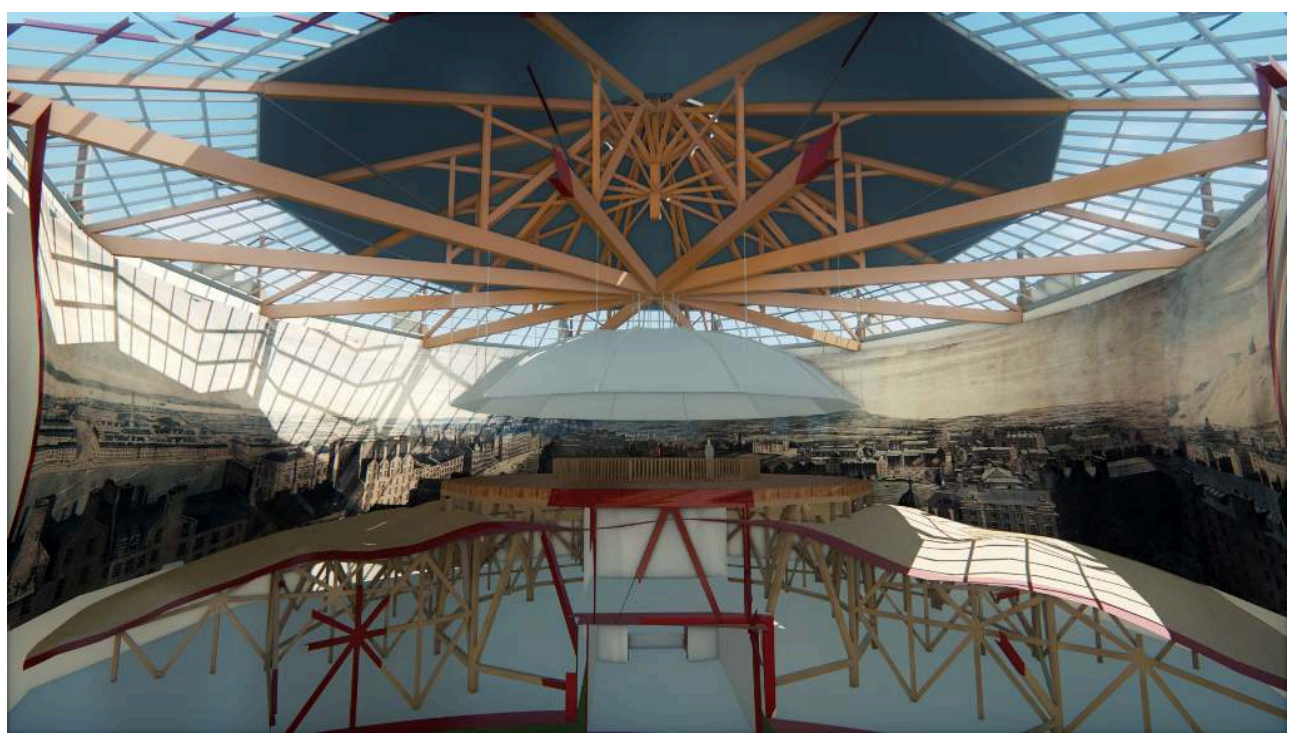

Modélisation 3D d'un panorama-type du xıx siècle à partir des plans dressés par Jacques Ignace Hittorff (1792-1867) dans son ouvrage Description de la Rotonde des panoramas élevée dans les Champs-Élysées (1842).

Image : Laurent Lescop.

4 La cohérence entre la représentation en tant que discours accompagnant une figuration et le support de cette figuration est l'enjeu même des dispositifs immersifs. 
Le premier de ces dispositifs qu'il est possible de reconnaître comme immersif date de la fin du XVIII ${ }^{e}$ siècle. Il s'agit du panorama inventé par Robert Barker en 1787, qui se présente comme une immense toile peinte déployée sur les parois d'un cylindre au centre duquel se trouve une plateforme accueillant le public. On objectera que d'autres dispositifs, telles les grottes ornées, peuvent être considérés comme antérieurs. Mais les panoramas ont la particularité de faire l'objet de brevets - c'est notamment le cas de celui de Robert Fulton datant de 1799 - décrivant clairement leur principe et les effets recherchés ${ }^{5}$ : ce sont des machines à illusion qui, par des jeux de circulations et de lumières, procurent un émerveillement lié à la découverte d'un paysage perçu à $360^{\circ}$. Les panoramas vont constituer au cours du $\mathrm{XIX}^{\mathrm{e}}$ siècle des attractions extrêmement prisées, couvrant de larges domaines allant de la découverte paysagère à la fresque historique $^{6}$. Ce qui est fascinant est leur capacité à incorporer les nouvelles technologies à mesure de leur découverte, que ce soit la photographie, la possibilité de diffuser du son ou encore la possibilité de créer des mouvements simulant un bateau en mer. À l'Exposition universelle de 1900, trois remarquables propositions immersives sont présentées au public : le Méréorama ${ }^{7}$ crée l'illusion d'un véritable voyage en bateau, le Cinéorama offre aux spectateurs d'expérimenter un voyage virtuel en ballon, et enfin le Transsibérien est un voyage immersif à travers l'Europe imaginé par l'architecte Georges Chedanne.

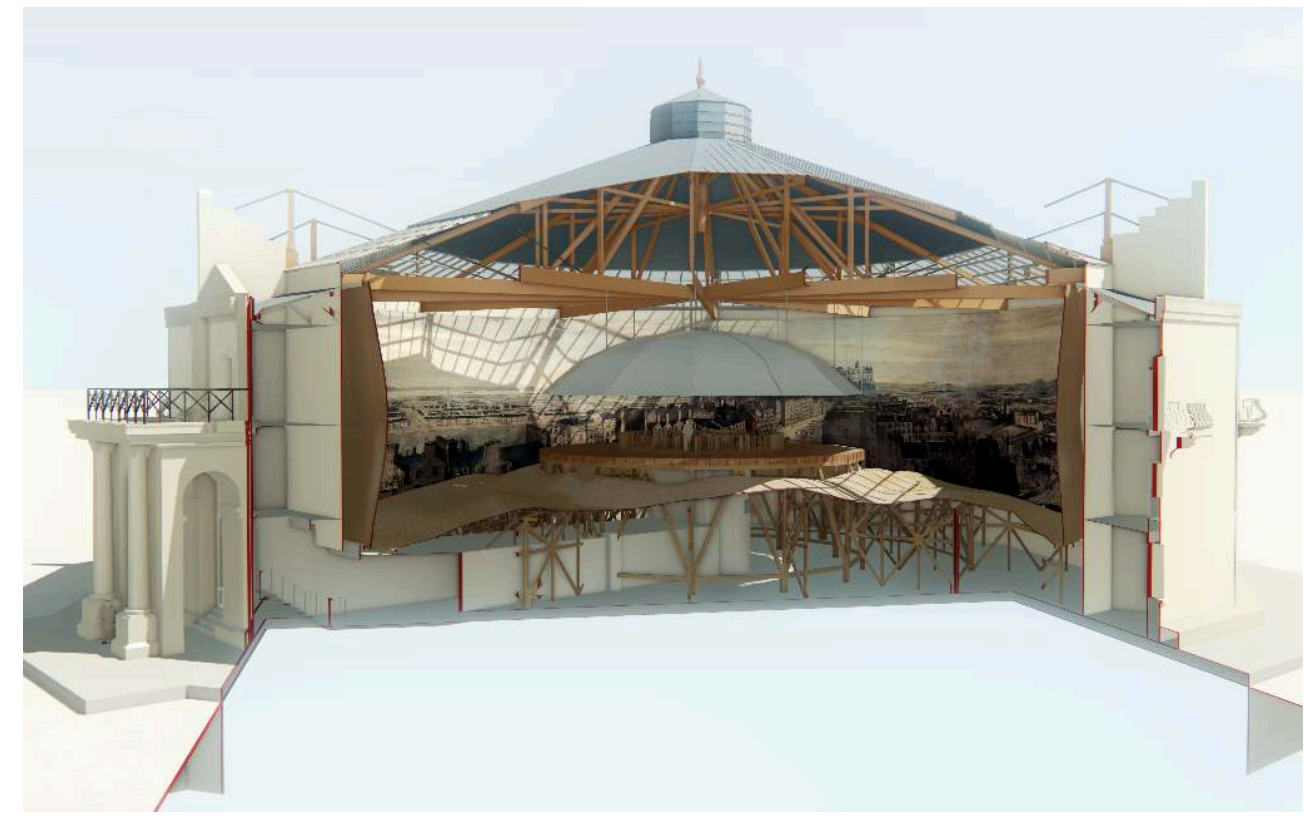

Modélisation 3D d'un panorama-type du xıx siècle à partir des plans dressés par Jacques Ignace Hittorff (1792-1867) dans son ouvrage Description de la Rotonde des panoramas élevée dans les Champs-Élysées (1842).

Image : Laurent Lescop.

5 Toutefois, les panoramas ne résistent pas à la nouvelle attraction des frères Lumière. Le cinéma, inventé en 1895 , connaît un succès fulgurant, passant en quelques années d'une attraction de foire à une puissante industrie. Le cinéma n'en cherche pas moins à renforcer de diverses manières son effet immersif. De nombreuses tentatives cinématographiques sont notamment mises en œuvre pour recréer l'impression immersive du panorama ${ }^{8}$, parmi lesquelles les trois écrans du Napoléon d'Abel Gance, en 1927, et le Circorama de Walt Disney, qui à partir de 1955 plonge ses visiteurs au cœur d'une couronne de onze écrans9. 
De nos jours, chacun est capable de produire à peu de frais son propre panorama. Les caméras spécialisées et les casques pour être immergé ne valent que quelques euros. Une aubaine pour la médiation, dont s'emparent déjà les grands musées et les centres d'interprétation comme celui de Lascaux, en Dordogne. Qu'en est-il toutefois de l'expérience collective? En 1889, Thomas Edison inventa le kinétoscope, une sorte de caisse verticale dans laquelle un - voire deux - spectateur visualisait un film. En 1894, William K. L. Dickson et Herman Casler proposèrent le mutoscope, sorte de paire de jumelles à travers laquelle l'utilisateur visionnait un carrousel d'images fichées dans un moyeu qu'il entraînait rapidement. Sous le nom kinora, les frères Lumière mirent au point un système presque similaire. Aucune de ces inventions ne se maintint face à l'expérience collective qu'offrait le cinéma. La même histoire se répète aujourd'hui entre les systèmes individuels et les dispositifs collectifs.

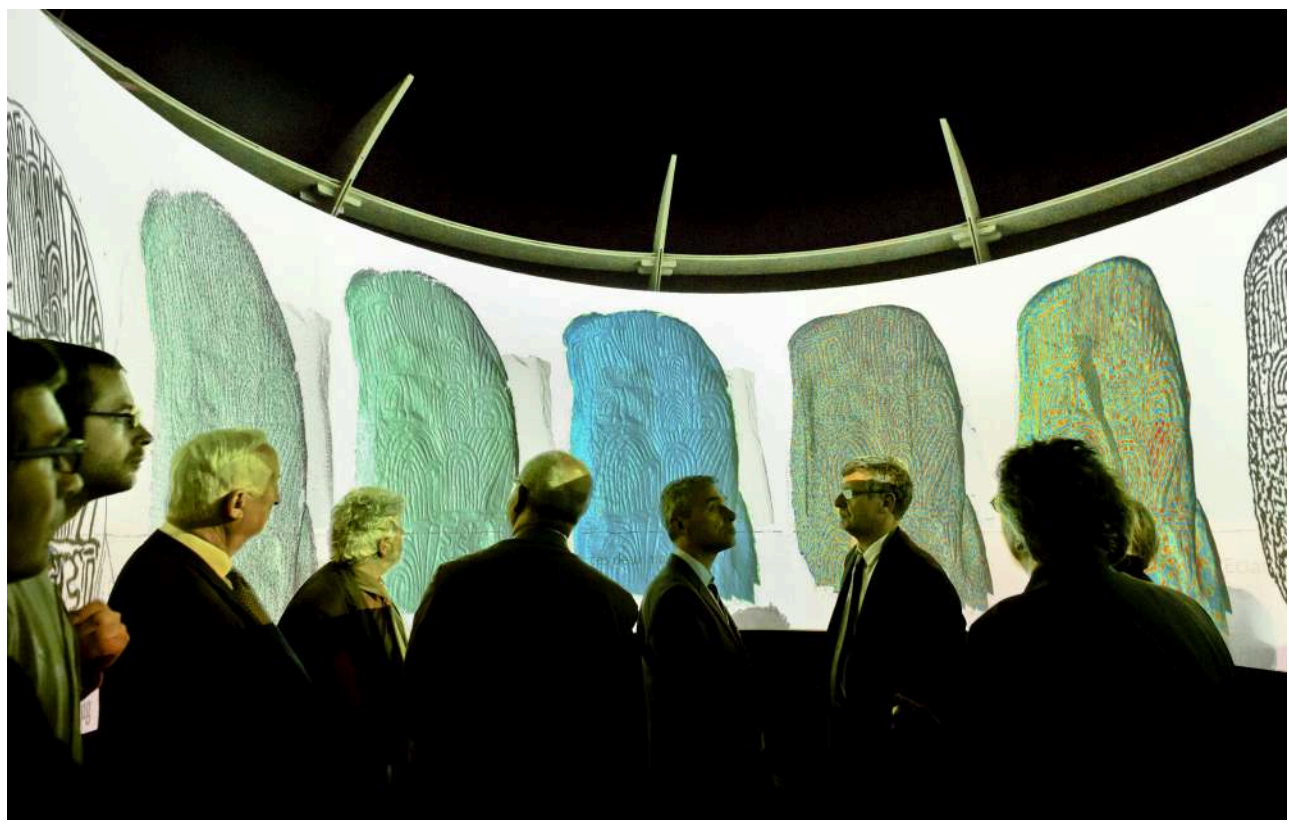

Découverte des stèles de Gavrinis (Morbihan) au moyen du dispositif immersif nomade Naexus.

Photo : Laurent Lescop.

\section{Unités de lieux et de temps}

7 L'immersion est souvent de nos jours assimilée à des expériences de réalité virtuelle dans lesquelles le spectateur vit, en temps réel et avec la possibilité d'influer par ses actions, l'expérience d'un ailleurs. Si une telle définition convient pour cerner rapidement une situation aisément saisissable, elle s'impose aux dépens de subtilités qu'il convient d'éclairer. Il est assez commun de comprendre une image comme un instant figé décrivant une unité de lieu, une unité de temps et une unité d'action. Cette habitude pousse à interpréter de cette façon les images qui se présentent à nous. Toutefois, comme l'a montré Frances Yates ${ }^{10}$, on trouve, au moins jusqu'au Quattrocento, des œuvres dans lesquelles plusieurs temporalités se déroulent dans le même espace. C'est le cas des tableaux de Masaccio ou de Filippo Lippi par exemple. Mais c'est aussi le cas de certains panoramas bien plus récents. Dans La Bataille de Reichshoffen peint en 1881, Théophile Poilpot et Stephen Jacob déploient l'action sur l'espace continu de la toile. En tournant le regard, le spectateur fait défiler le temps. 
Dans le même ordre d'idée, le film animé Pearl de Patrick Osborne, tourné à $360^{\circ}$ en 2017, met en scène une histoire se déroulant dans une voiture ${ }^{11}$. Selon la direction du regard du spectateur, la narration prend des chemins différents, induite par des temporalités localisées dans une direction ou dans une autre. En outre, le temps n'est pas nécessairement défilant : il peut être condensé sur l'espace de la toile comme le $\mathrm{Nu}$ descendant un escalier que Marcel Duchamp a présenté en 1912 ou, dans le cas des panoramas et des films à $360^{\circ}$, concentré à la surface d'une sphère ou d'un cylindre.

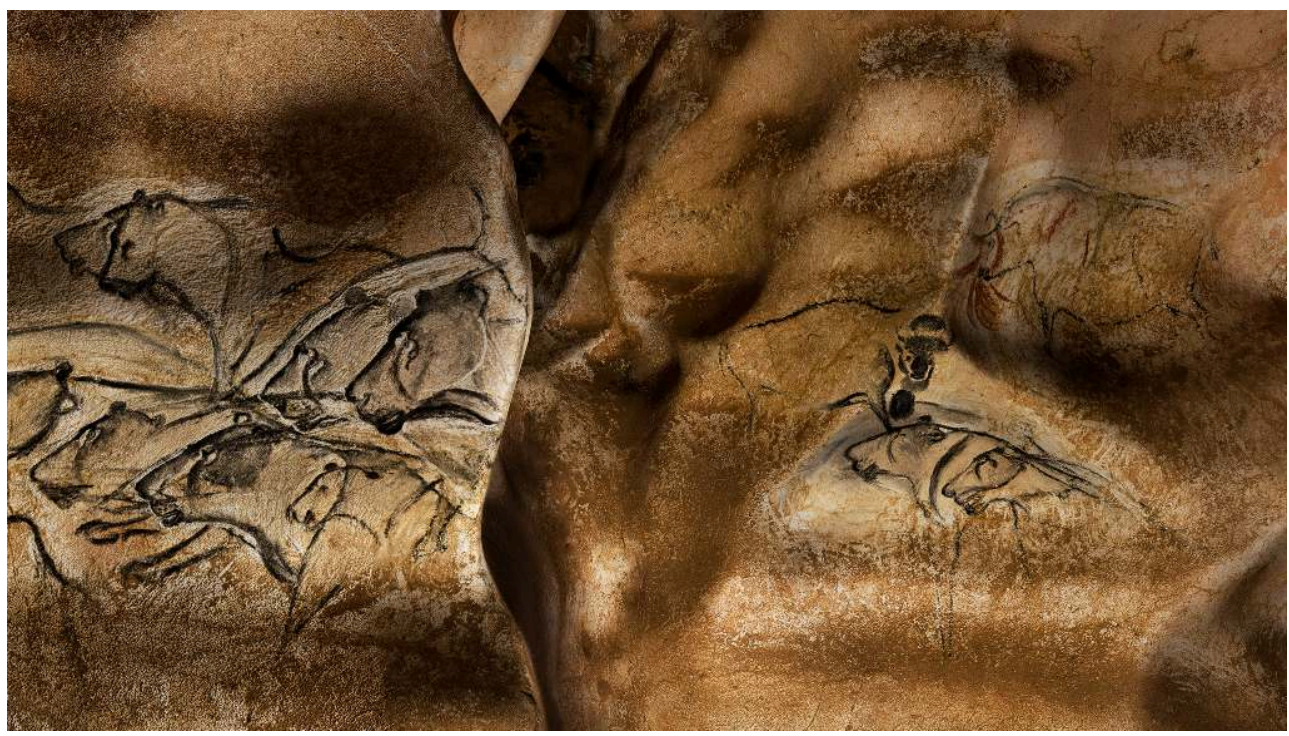

Parois ornées de la Salle du Fond de la grotte Chauvet-Pont d'Arc. Photogramme extrait du film Le Dernier Passage de Pascal Magontier et Jean-Michel Geneste, réalisé à partir du modèle 3D de la grotte.

Modélisation 3D : Perazio Engineering / éclairage : Hugo Barbier / cliché : Rup'art productions.

De même dans les grottes ornées, il n'est pas exclu que le temps se trouve entre deux figures se faisant face. La médiation des figures pariétales se fait essentiellement par la photographie et le film. Ces deux médiums imposent un cadre au moyen duquel, inévitablement, un sujet ou un groupe de figures se trouve arraché de l'environnement complexe formé par la cavité et son système orné - contribuant à percevoir la grotte comme une sorte de galerie sauvage que le temps aurait rouverte pour nous. Cette détermination technique a deux conséquences : découper et isoler arbitrairement des figures potentiellement liées les unes aux autres, et gommer les éléments contextuels porteurs d'indications d'échelle, de disposition et d'interrelations. Dans ce contexte, l'immersion et le regard à $360^{\circ}$ en tant que support d'exploration dynamique et non dirigée redonnent des correspondances, restituent le contexte, donnent à lire le temps qui existe entre deux figures. On peut cependant s'interroger sur la place laissée ici à l'imaginaire du spectateur. C'est en effet dans le hors-champ que se construit l'imaginaire qui accompagne les images cadrées. Dans une narration à $360^{\circ}$, où rien n'échappe au regard, où tout semble défini, l'imaginaire n'est-il pas exclu? Loin de cela, on fait le constat que le champ et le hors-champ se reconstruisent par l'espace, par des masques placés à certains endroits, par l'obscurité qui vient creuser des profondeurs insondables, par la distance enfin qui renvoie l'imaginaire au-delà des limites du visible. C'est qui précisément est mis en scène dans Le Dernier Passage. 


\section{Narration}

Le Dernier Passage ${ }^{12}$ est un long travelling en plan séquence au sein de la grotte Chauvet. D'un mode encore cinématographiquement classique, ce film en relief, conçu pour épouser le rythme du pas humain, pourrait être naturellement transposé pour une visualisation à $360^{\circ}$. La polysémie du terme "passage" s'applique aussi bien à la géographie de la grotte qu'à l'acte de cheminer une dernière fois en ce lieu que la fréquentation condamne. Le passage c'est la transition, c'est la transgression. Le dernier passage est la transgression du visiteur virtuel et contemplatif dans la caverne oubliée. La narration est affaire de passage, comme l'est plus encore l'immersion, d'un état à l'autre. L'aller produit ce que le retour imprime, un changement profond et définitif.

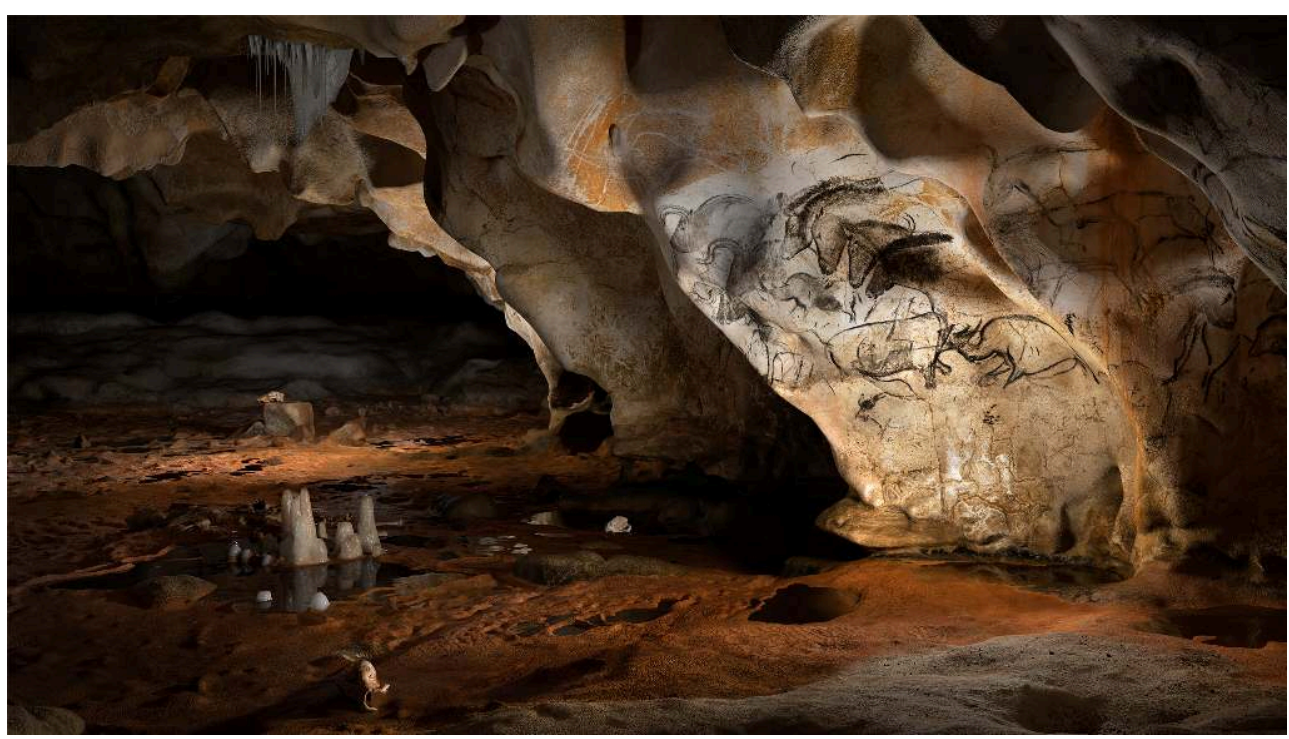

La Salle du Crâne de la grotte Chauvet-Pont d'Arc. Photogramme extrait du film Le Dernier Passage de Pascal Magontier et Jean-Michel Geneste, réalisé à partir du modèle 3D de la grotte.

Modélisation 3D : Perazio Engineering / éclairage : Hugo Barbier / cliché : Rup'art productions.

Concevoir une histoire, c'est planifier pour le lecteur ou le spectateur un jeu de promesses et de récompenses. Les promesses concernent épreuves et découvertes, les récompenses sont des accomplissements et des dévoilements. Pour le spectateur, le fil ne doit pas se sentir, bien au contraire, surtout dans une narration immersive où le mouvement et certaines interactions sont possibles, où de multiples chemins narratifs doivent se dessiner, s'entrevoir, comme dans la nouvelle de Jorge Luis Borges Le Jardin aux sentiers qui bifurquent ${ }^{13}$. Toutefois, l'infinité narrative reste une illusion, et le fil tire bien le spectateur d'un point à un autre dans un ordonnancement maîtrisé. L'auteur et réalisatrice Jessica Brillhart ${ }^{14}$ développe le concept de "probabilistic experiential editing " («montage expérientiel probabiliste ») qui consiste à placer dans le champ de vision des points d'intérêt qui invitent le spectateur à aller d'une étape à l'autre en laissant, entre les deux, un espace de liberté suffisant pour effacer de l'ensemble de l'expérience toute impression de déterminisme. Au montage séquentiel du cinéma se substitue en définitive un montage "conséquentiel » en immersion où s'enchaînent causes et effets autour de déclencheurs narratifs situés aux points d'intérêt. Lorsque l'on travaille à partir de Chauvet ou Lascaux à élaborer un cheminement à l'intérieur des galeries, il apparaît nettement que cette logique de points d'intérêt y est déjà à 
l'œuvre. Une fresque semble en désigner une autre, un événement géologique nous laisse penser qu'il introduit un thème tandis qu'un autre le poursuit. Cette dialectique entre le contexte et les figures construit une histoire cinétique qui inexorablement, conduit vers le thème majeur. Le réalisateur peut dès lors s'appuyer sur cette trame préexistante pour placer ses propres points d'intérêt. Il le fait par le biais de la lumière et du son spatialisé qui viendront, le moment opportun, induire la direction où regarder, à la façon d'un raccord de regard tel qu'on peut le faire dans un montage cinématographique classique. Dans le film My Brother's keeper ${ }^{15}$, écrit et réalisé par Connor Hair et Alex Meader, le choix a été fait de flouter les parties non déterminantes pour la narration, seule la zone à enjeu est nette. Une solution alternative consiste, dans le cadre d'une expérience immersive partagée, de laisser chacun accepter de voir et de manquer des événements. Comme dans le cinéma de Jacques Tati et particulièrement pour le film Playtime $^{16}$, le spectateur interpelle son voisin pour l'inviter à ne pas manquer un détail important ou insolite : le commentaire est autorisé, voulu, il porte la narration ${ }^{17}$.

11 La narration en immersion possède la troublante et stimulante propriété de multiplier les dimensions temporelles. L'idée du temps réel n'est que l'un des aspects de l'expérience proposée et, nous l'avons déjà entrevu, plusieurs temporalités peuvent exister dans le même espace. Dans les jeux vidéo ${ }^{18}$ par exemple, une heure ne dure que deux minutes tandis qu'une journée entière se déroule en quarante-huit minutes. Pourtant, tout semble se dérouler en temps réel. Il existe donc deux chronologies simultanées, celle de l'utilisateur et celle de l'espace diégétique. Cette possibilité d'associer plusieurs temporalités dans le même espace permet de jouer également de l'ellipse temporelle, figure majeure de la narration. Dans une histoire contée, on passe, pour en augmenter l'intérêt et l'intensité, du temps continu au temps discret. Cet instrument narratif ne semble pas disponible si l'on considère l'immersion comme une narration en temps réel. Là encore, il existe des solutions explorées par le monde vidéo ludique. Dans la série Assassin's Creed ${ }^{19}$, réputée pour la rigueur historique de ses restitutions y compris de la préhistoire, les espaces urbains narrativement les moins intéressants sont compressés, tandis que l'utilisateur pense se déplacer dans un continuum similaire à celui de l'expérience réelle - d'autant plus lorsqu'il connaît le référent réel de la ville dans laquelle il se déplace. L'ellipse narrative fait se rapprocher les points d'intérêt de telle façon que l'on peut déterminer que le temps a été converti en espace ${ }^{20}$.

12 La conversion du temps en espace concerne surtout les espaces extérieurs dans lesquels une multitude de repères temporels situent chaque moment dont le premier et le principal est bien entendu l'astre solaire. Dans le cas d'une compréhension phénoménologique de l'appropriation symbolique de surfaces extérieures ornées, il peut être important de poser le regard au bon instant afin de croiser l'intentionnalité graphique et les phénomènes naturels. Dans le cas des sites pariétaux situés dans les profondeurs des cavernes, la notion du temps devient tout autre. Tout spéléologue peut témoigner de cette sensation de suspension temporelle et nous avons encore en mémoire les expériences «hors du temps " menées par Michel Siffre ${ }^{21}$ qui ont commencé à décrire le fonctionnement du cycle circadien.

13 Dans le monde réel et dans le domaine de la médiation en archéologie, il existe un exemple construit de temps converti en espace. La Caverne du Pont d'Arc ${ }^{22}$, conçue par les architectes Vincent Speller et Xavier Fabre et ouverte en 2015, propose 
d'expérimenter l'espace réel de la grotte à l'échelle 1, mais replié, densifié, rempli d'ellipses narratives qui imposent un rythme à la visite. Les archéologues et géomorphologues de l'équipe de recherche ont eux-mêmes sélectionné et conçu les séquences narratives retenues, et déterminé ces ellipses afin que le rythme final soit similaire à celui de la grotte initiale ${ }^{23}$. Comme l'aurait fait un scénariste pour une visite immersive en images, les architectes ont transformé le temps en espace pour augmenter, par compression émotionnelle, la sidération des visiteurs.

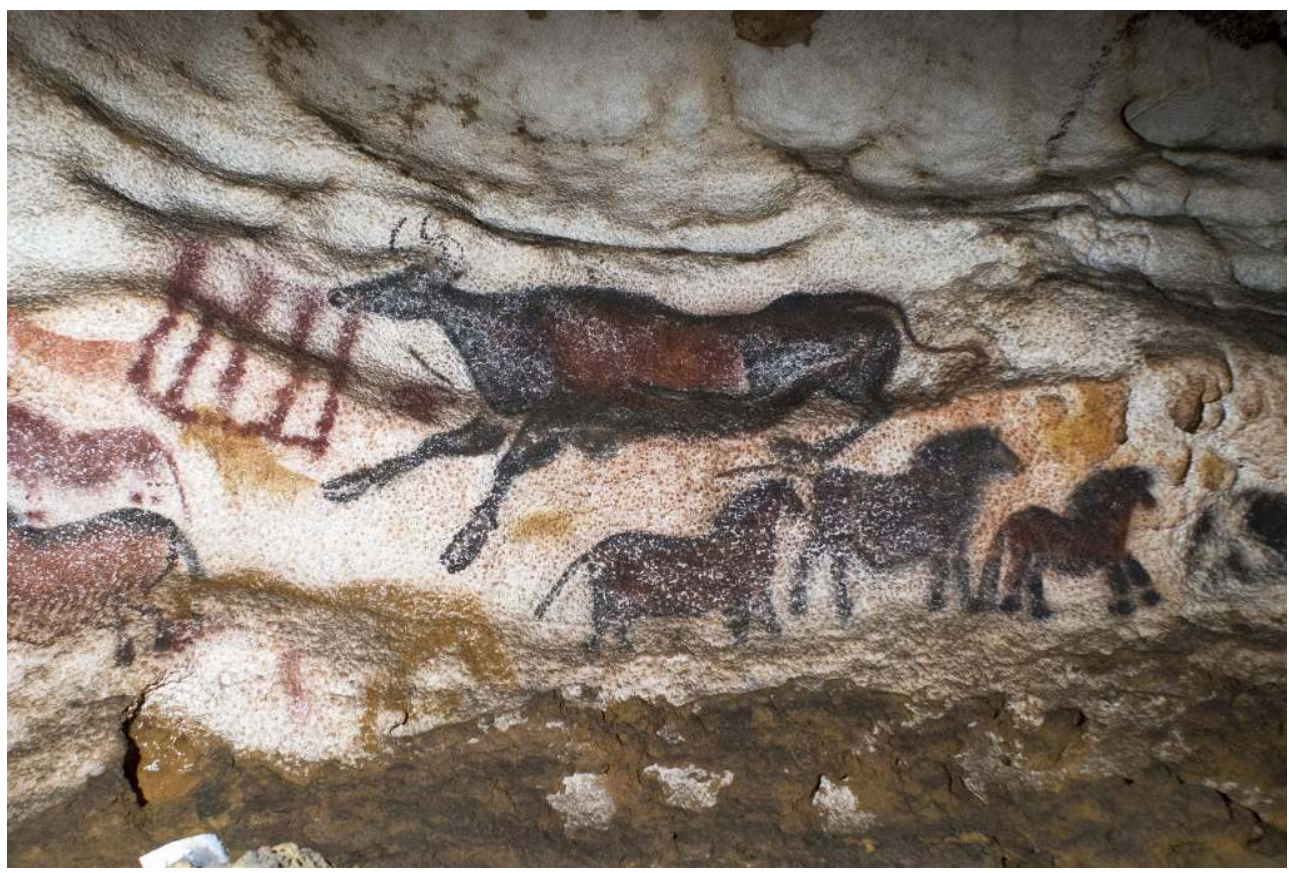

Parois ornées de la grotte de Lascaux.

Photo : Jean-Michel Geneste / cliché : Centre national de préhistoire, ministère de la Culture.

\section{Le point de vue}

L'immersion à $360^{\circ}$ cherche à plonger le spectateur dans l'espace du récit, à lui faire passer ce que le scénographe Josef Svoboda appelait la «ligne de partage des eaux ». Dans la médiation historique, archéologique, l'utilisation des techniques immersives implique de s'interroger sur le point de vue que cherche à développer la narration et donc sur la place à donner au spectateur dans la visite qui lui est proposée. À un premier niveau, une alternative se présente: celle du visiteur temporel ou celle de l'incarnation d'une personne vivant à l'époque de la restitution proposée. L'enjeu est bien entendu l'empathie que l'on souhaite voir se développer avec le sujet. Il existe plusieurs façons de concevoir une incarnation dans la narration immersive à $360^{\circ}$. La première est celle où le corps du spectateur est un fantôme, il n'a pas de matérialité et a donc potentiellement la capacité de se poser n'importe où ; le spectateur est invisible aux protagonistes des scènes qu'il visualise. La deuxième présente une incarnation partielle : le point de vue est cohérent avec la posture d'une personne présente dans la scène et les personnages peuvent interagir avec elle. La troisième forme est celle où un corps est partiellement visible - ce sont généralement les bras, les jambes et le tronc qui sont dévoilés lorsque bouge la tête. Le réalisateur Guy Shelmerdine en joue dans deux de ses œuvres, Catatonic ${ }^{24}$ et $\mathrm{Mule}^{25}$ : le spectateur prend la même pose que le 
personnage virtuel qu'il incarne et se voit subir les pires outrages jusqu'à la mort par crémation. Un principe similaire est proposé dans les applications pornographiques ${ }^{26}$ : le spectateur prend la position du sujet qu'il incarne mais pour cette fois recevoir des traitements bien moins traumatiques. Habiter le corps de l'autre pour activer l'empathie est un argument fort pour les réalisateurs à $360^{\circ}$. C'est ainsi que Alejandro Gonzalez Iñárritu dans Carne y arena ${ }^{27}$, présenté à Cannes en 2017, place son public dans la peau d'un migrant mexicain. Notes on blindness raconte, sous la forme d'un film classique $^{28}$ et d'une application VR immersive ${ }^{29}$, la perte progressive de la vision par le théologien John Hull. Plus étonnante est l'expérience générée par le laboratoire BeAnotherLab ${ }^{30}$ appelée "The Machine to be another ${ }^{31}$ ", et plus précisément l'application Gender Swap dans laquelle l'utilisateur change de sexe.

Pour le moment, il n'est pas beaucoup d'applications immersives proposant de se mettre dans la peau d'un homme ou d'une femme du mésolithique, si ce n'est dans quelques applications artistiques ou des jeux vidéo tels Far Cry Primal ${ }^{32}$ ou Wild ${ }^{33}$. Dès lors quelle serait la représentation à proposer? Nous l'avons évoqué plus haut, le système de représentation proposé par nos outils est celle qui s'est imposée depuis l'occident au reste du monde. Cette hégémonie laisse facilement pensée qu'elle est naturelle et il est vrai que la confusion entre le réel et le virtuel valide cette hypothèse. Toutefois, alors que pour l'étude de figures issues de temps anciens, nous sommes déjà dans la représentation de représentation, dans un méta discours dont l'objet même échappe, les enjeux d'une empathie immersive ne peut être évacuée d'un revers de main.

\section{La métaphore}

Une façon d'échapper à cette incorporation qui pose plus de questions qu'elle n'en résout pourrait être de revenir à l'expérience collective telle qu'évoquée plus haut et d'utiliser, comme dans les panoramas de l'Exposition universelle de 1900, un véhicule pour transporter les spectateurs en un ailleurs distant. Un véhicule, étymologiquement, est ce qui sert à transporter, mais également à transmettre. Un train, par exemple, utilisé comme dispositif immersif pour la visite de sites historiques, est à la fois l'analogie du véhicule transportant ses visiteurs et la métaphore de la transmission de la connaissance ou de l'émotion. Un train, transmetteur et transporteur, se définit selon différents modes : la métaphore, l'allégorie et l'évocation. La métaphore vient du mot grec «metaphorá » qui signifie transport, c'est une figure de style qui permet de désigner un objet par un autre objet, lui prêtant ainsi certaines qualités ou caractéristiques. Le contexte permet de comprendre le transfert de sens d'un objet à l'autre. Dans le cas d'un train dédié à un voyage immersif, la métaphore est celle d'un train réel marqué par les caractéristiques architecturales et fonctionnelles qui le définissent: un wagon aux proportions correctes, des bancs, de larges baies. L'évocation permet de faire exister une idée ou un objet, de l'activer afin de le rendre présent chez chacun. Ici, l'évocation première est celle du voyage qui permet de rendre effectif, une expérience purement illusionniste dans laquelle la fonction conative des spectateurs-passagers est sollicitée.

En quoi cela est-il important pour une narration à $360^{\circ}$ dédiée à l'archéologie ? De fait, cela induit les choix artistiques et techniques de la proposition. La métaphore, l'allégorie ou l'évocation d'un voyage suppose deux figures géométriques : la ligne ou le 
cercle, répondant par ailleurs à deux conceptions de l'existence telles qu'on les retrouve dans l'ensemble des cultures. La ligne implique que le point de départ et d'arrivée ne sont pas les mêmes, le cercle impose le contraire. L'une et l'autre figure portent également la même alternative: celle du changement ou celle de la conservation. Le voyage peut nous mener ailleurs sans qu'il ne nous change, le voyage peut nous ramener à notre point de départ et nous sommes métamorphosés - pour évoquer deux des quatre possibilités. Il existe encore une autre figure, celle de la ligne avec retour par le même chemin qui est l'une des figures de plusieurs mythes et récits de retour. Dans le film Le Dernier Passage, la figure proposée est celle de la ligne qui aboutit au grand panneau aux lions comme acmé de la visite : la vision est si puissante qu'il est difficile d'en sortir. S'en détacher et revenir, c'est changer d'état, c'est transgresser. Le Dernier Passage donne à vivre l'expérience physique du noir des grottes, un noir absolu, un noir d'aveugle, comme un passage nécessaire pour ceux qui veulent s'aventurer dans le monde souterrain. L'œuvre-grotte ne peut se découvrir vraiment que porteur d'une faible lumière, les yeux écarquillés et les oreilles aux aguets. La fin du film est construite en symétrie. La vision, si puissante soit-elle, est rendue à l'obscurité. Le chemin du retour passe par le noir absolu. Ce noir d'aveugle est désormais peuplé par des images mentales, qui ne sont pas forcément identiques aux œuvres pariétales, mais qui toutes sont produites par l'expérience de la grotte. C'est toute la symbolique d'un voyage initiatique dans un cas ou d'une conquête exploratoire dans l'autre. Initiés, nous revenons chargés de nouvelles connaissances, nous pouvons dès lors explorer de nouveaux horizons de connaissances.

\section{La conquête du beau}

Dans Nus et Paysages, Alain Roger montre que la beauté favorise l'appropriation, que le beau donne à aimer. C'est ce qu'il appelle l'artialisation ${ }^{34}$ : il faut rendre le sujet d'étude désirable, non seulement pour se délecter de sa vision, mais également pour le comprendre et se l'approprier. Grâce à cela, on franchit le seuil de l'indéfini pour aller vers un ensemble composé, la connaissance. La tendance actuelle est de déléguer aux outils numériques cette mission d'artialisation. Présenté comme une nouvelle rupture culturelle semblable à celle de l'invention de l'écriture, le numérique interroge les pratiques classiques, les ringardisant parfois. Toutefois, il s'agit de rester prudent : en rendant belle la pratique marchande, les applications informatiques dédiées au patrimoine font trop souvent passer la vente pour de l'expression artistique ou une posture inscrite dans la mode.

Les questions évoquées ici visent à approcher les enjeux d'une narration à $360^{\circ}$ dédiée à l'archéologie. Nous avons vu que les dispositifs immersifs s'inscrivent dans une histoire qui commence au cœur des Lumières, alors que se construisent les notions de paysage, de nation et d'identité culturell ${ }^{35}$, d'ethnologie et d'archéologie. Les technologies les plus récentes viennent servir l'immersion dans le patrimoine historique et contemporain. Les dispositifs se diversifient et une lutte s'engage entre ceux proposant une expérience individuelle contre ceux offrant une expérience collective, ces derniers finissant par l'emporter. Un siècle plus tard, de nouvelles techniques rejouent le combat avec probablement la même issue à terme, et un siècle plus tard, le terme même de réalité virtuelle se réinvente. En effet, contrairement à ce qui communément admis, son origine ne se trouve pas chez Myron Krueger, ni même Antonin Artaud 
comme il est parfois indiqué, mais chez Joseph Willm dont la citation suivante semble destinée à notre sujet ${ }^{36}:$ "Dans le système de Fichte, le moi n'est plus une simple faculté de sentir et de penser, un simple sujet, subissant l'action des choses selon sa nature, et réagissant sur elles par la pensée : il est virtuellement tout la réalité, et travaille à la produire pour soi, à s'en donner la conscience actuelle. De son côté, le non-moi est en soi vide de toute réalité ; ce n'est que la réalité virtuelle du moi, considéré comme posée hors de lui, et il ne se réalise véritablement qu'autant qu'il est posé dans le moi, qu'il est déterminé par le sujet ${ }^{37}$. " L'immersion et la narration à $360^{\circ}$ ne créent pas seulement l'illusion d'entrer dans l'espace diégétique, elles parviennent à faire coexister plusieurs temporalités dans le même espace, réfutant ainsi la correspondance littérale réel/virtuel. Mais cette immersion attise plus spécifiquement encore les enjeux du point de vue lorsque l'on joue à s'incorporer dans l'acteur de l'espace des récits. Une façon de s'en dégager et de retrouver la communion d'une expérience collective serait, comme le faisaient les panoramas de jadis, d'user d'un véhicule métaphorique pour conduire passagers et connaissances. Le voyage proposé est à la fois une découverte et une élévation, une possibilité d'empathie et de distinction.

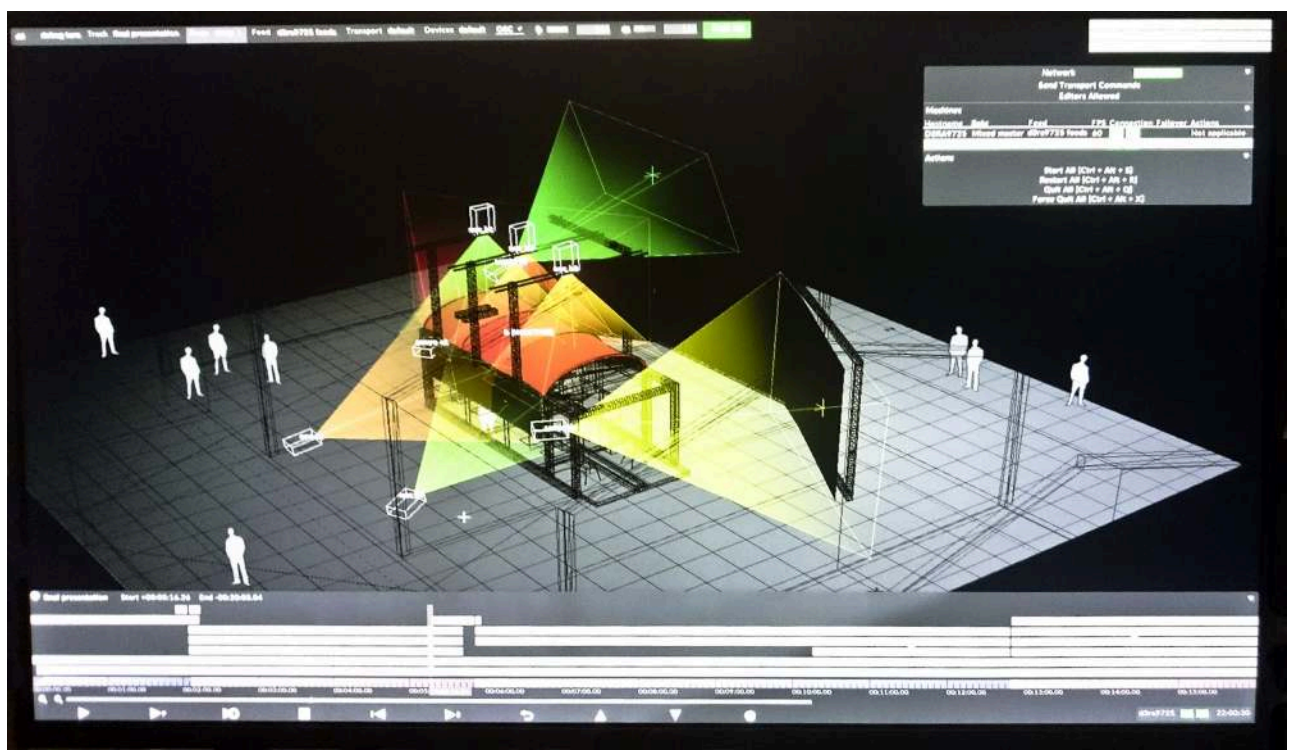

Conception du dispositif Erodor, dispositif immersif mobile pour la valorisation des œuvres pariétales (capture d'écran).

Photo : Laurent Lescop.

Le dispositif Erodor, actuellement à l'étude, contient l'ensemble de ces enjeux. Il parie sur un voyage métaphorique à la découverte du monde pariétal. Assis face à face, les voyageurs-spectateurs placés au centre de l'image traverseront les paysages de l'Alberta, de la Namibie et de l'Australie et plongeront dans les profondeurs de Lascaux ou de Chauvet. Dans la maquette réalisée pour les premiers tests, un vénérable monsieur de cent un ans faisait face à un bambin de six ans. Durant ce voyage initial, leurs regards se sont plusieurs fois croisés tandis qu'ils tentaient de comprendre tout ce qui se déroulait autour d'eux. À un moment, ils se sont regardés l'un l'autre et ont échangé un large sourire complice. Ils étaient heureux, ils vivaient ensemble une expérience inoubliable... 


\section{NOTES}

1. PICON Antoine, Culture numérique et architecture. Une introduction, Bâle, Birkhäuser, 2010.

2. CORMERAIS Franck, "L'imaginaire en fabrication d'une ville contributive, Nantes", in CORMERAIS Franck \& GILBERT Jacques (dir.), Poétique(s) du numérique, vol.3, Imaginaire et nouvelles scènes des villes, Lavérune, L'Entretemps éditions, 2015, p. 11-14.

CR\%20conf\%C3\%A9rence\%20Kalist\%C3\%A9\%20Et\%C3\%A9\%202011_ALTRAN\%281\%29.pdf

4. LESCOP Laurent \& LU Yang, "Les dimensions de la perspective: quand les modes de représentations européennes et chinoises rencontrent l'image numérique ", intervention au cours du $8^{\mathrm{e}}$ séminaire de conception architecturale numérique SCAN'18 (Nantes, octobre 2018).

5. BAPST Germain, Essai sur l'histoire des panoramas et des dioramas, Paris, Imprimerie nationale /

G. Masson, 1891.

6. ROBICHON François, "Les Panoramas en France au XIX ${ }^{\mathrm{e}}$ siècle ", thèse d'art et archéologie, Nanterre, université Paris-Nanterre, 1982.

7. www.worldfairs.info

8. MICHAUX Emmanuelle, Du panorama pictural au cinéma circulaire. Origines et histoire d'un autre cinéma, 1785-1998, Paris, L'Harmattan, 1999.

9. http://www.waltdisney.org/blog/plussing-disneyland-1955

10. YATES Frances, L'Art de la mémoire, Paris, Gallimard, coll. «Bibliothèque des histoires », 1975 [1966].

11. Ce film a été primé d'un Emmy Award pour «Outstanding innovation in interactive storytelling ».

12. MAGONTIER Pascal, Le Dernier Passage, texte de Jean-Michel Geneste, France, Rup'Art Productions / Perazio Engineering, 2015, 28 min.

13. BORGES Jorge Luis, Fictions, Paris, Gallimard, coll. « Folio », 1983 [1951].

14. http://filmmakermagazine.com/96090-look-into-the-cut/

15. HAIR Connor \& MEADER Alex, My Brother's keeper, USA, StoryTech Immersive / Perception Squared / Technicolor Experience Center / PBS Digital Studios, 2017, 11 min.

16. TATI Jacques, Playtime, France / Italie, Specta Films / Jolly Films, 1967, 124 min.

17. GUÉRAND Jean-Philippe, Jacques Tati, Paris, Gallimard, coll. « Folio », 2007.

18. Comme dans Grand Theft Auto dont la version III, en 3D à la troisième personne, est sortie en 2001 (développé par Rockstar North et Rockstar Games).

19. Développée par Ubisoft depuis 2004.

20. LESCOP Laurent, "Narrative grammar in 360 », intervention au cours de l'International Symposium on Mixed and Augmented Reality (ISMAR) organisé par IEEE Computer Society Technical \& Conference Activities Board (Nantes, France, 9-13 octobre 2017), IEEE International Symposium on Mixed and Augmented Reality Adjunct Proceedings, 2017. Disponible en ligne, https://www.researchgate.net/publication/320403732_Narrative_Grammar_in_360 [lien valide en mars 2020].

21. SIFFRE Michel, Hors du temps. L'expérience du 16 juillet 1962 au fond du gouffre de Scarasson par celui qui l'a vécue, Paris, Julliard, 1963.

22. Initialement dénommé « Caverne du Pont d'Arc », le lieu a été renommé " Grotte Chauvet 2 Ardèche » en février 2019.

23. GENESTE Jean-Michel, DELANNOY Jean-Jacques \& TOSELLO Gilles, « De la grotte à la caverne et de l'archéologie au public », Les Nouvelles de l'archéologie, n 147, " Musées d'archéologie au début du XXI ${ }^{\mathrm{e}}$ siècle », 2017, p. 39-44. 
24. SHELMERDINE Guy, Catatonic, USA, Dark Corner Studios / Here Be Dragons production, 2015, $5 \mathrm{~min}$. Voir https://www.guyshelmerdine.com/virtual-reality [lien valide en mars 2020].

25. SHELMERDINE Guy, Mule, USA, Dark Corner Studios, 2015, 6 min. Voir https:// www.guyshelmerdine.com/virtual-reality [lien valide en mars 2020].

26. Qu'il s'agira de retrouver par soi-même !

27. IÑÁRRITU Alejandro Gonzalez, Carne y arena, USA, Legendary Pictures, 2017, 7 min.

28. MIDDLETON Peter \& SPINNEY James, Notes on blindness, Royaume-Uni / France, Archer's Mark / Agat Films \& Cie / Arte France, 2016, 90 min.

29. MIDDLETON Peter \& SPINNEY James, Notes on blindness, France / Royaume-Uni, Agat Films \& Cie / Ex Nihilo, 2016, 7 min.

30. http://beanotherlab.org/

31. http://www.themachinetobeanother.org/

32. Conçu par Dan Hay, édité par Ubisoft, 2016.

33. Conçu par Michel Ancel, édité par Sony Interactive Entertainment, 2018.

34. ROGER Alain, Nus et Paysages. Essai sur la fonction de l'art, Paris, Aubier, 2001 [1978].

35. THIESSE Anne-Marie, La Création des identités nationales. Europe XVIII ${ }^{e}-\mathrm{XX}$ siècle, Paris, Éditions du Seuil, coll. « L'univers historique », 1999.

36. LESCOP Laurent, «Topologies de l'immersion », Études digitales, $n^{\circ} 4$, vol. 2, «Immersion », 2019, pp.21-52.

37. WILLM Joseph, Histoire de la philosophie allemande depuis Kant jusqu'à Hegel, vol. 2, Paris, Ladrange, 1847, p. 345.

\section{RÉSUMÉS}

Les outils de relevé numériques, prolongés par les techniques de modélisation, de mise en lumière et d'animation, permettent de restituer des environnements et des objets soit partiellement dégradés, soit inaccessibles, avec une grande précision géométrique. Pour le chercheur, c'est une aide à la contextualisation, pour le public, c'est la possibilité d'avoir accès à des sites parfois distants ou d'accès limité, et de découvrir de l'information déjà interprétée.

Cet article cherche à identifier les enjeux de médiation de cette production scientifique. La médiation de ces environnements en 3D pose incidemment, dans un premier temps, la question $\mathrm{du}$ dispositif de présentation, puis dans un deuxième temps du contexte et des environnements de présentation. Notre propos interroge donc, en le référençant dans l'histoire des technologies immersives, l'articulation entre le fond présenté et la forme de la structure, et ce bien au-delà de la seule considération scénographique.

Parmi les dispositifs de médiation, les dispositifs immersifs offrent les enjeux les plus stimulants. Considérés comme les derniers nés des technologies numériques, ils sont en fait les héritiers d'une longue généalogie passant par le cinéma multi-écran, les planétariums, les panoramas, les coupoles peintes et peut-être, quelques exemples de peintures pariétales. Aujourd'hui, les dispositifs immersifs donnent à expérimenter les sites archéologiques dans leur contexte, qu'ils soient à ciel ouvert ou dans des espaces clos. Leur conception fait d'emblée surgir des enjeux narratifs : la temporalité choisie, la gestion des données lacunaires, l'insertion de scènes du vivant, le traitement des ambiances. 
La pratique montre que pour répondre à ces enjeux narratifs on ne saurait se contenter, en contexte immersif, de recourir à la grammaire visuelle ou filmique classique. Il convient de développer une grammaire narrative à $360^{\circ}$, une grammaire proprement immersive. En partant d'une réalisation concrète en cours de développement, liée à l'étude pariétale, et d'un corpus de références allant des panoramas aux outils numériques, nous décrirons donc les constituants de cette grammaire narrative à $360^{\circ}$ et essayerons de montrer comment dès lors la forme construite d'un dispositif peut être en cohérence avec le contenu narratif. Cette réflexion pousse la représentation en archéologie au-delà de la visualisation descriptive et questionne les relations entre les discours produits et leurs transcriptions visuelles.

Digital documentation tools, helped by modelling, underscoring and animation techniques, allow us to recreate environments and objects that may be either partially degraded or inaccessible, with great geometric precision. This helps researchers with contextualisation, and for the public, this is an opportunity to access distant sites or sites with limited access, and to discover information that has already been interpreted.

One of the first questions related to the 3D mediation of these environments is the matter of the display device. It may be a generic device, but more and more specific devices are being developed, raising many questions about the articulation between the content being displayed and the shape of the structure, way beyond the mere scenographic consideration.

of all these devices, the immersive devices offer the most interesting content. They let us experience the archaeological sites with their full context, whether they are outdoors or in enclosed spaces. Their conception immediately underlines narrative issues: chosen temporality, the processing of insufficient data, the insertion of live scenes and the treatment of atmospheres. Practice shows that, to solve those narrative issues in an immersive context, the classical visual or filmic grammar will not be sufficient. We must develop a $360^{\circ}$ narrative grammar, a properly immersive grammar. By starting from a concrete realisation, currently in development, linked to the study of parietal arts and a corpus of references ranging from panoramas to digital tools, we will describe the constitutive parts of that $360^{\circ}$ narrative grammar that pushes the archaeological representation beyond the descriptive visualisation and questions the relationship between the produced discourse and its visual transcription.

\section{INDEX}

Mots-clés : 3D, archéologie, immersion, modélisation, relevés numériques, restitution

Keywords : immersion, 3D, archaeology, modelling, digital documentation, digital restitution

\section{AUTEURS}

\section{LAURENT LESCOP}

Architecte, professeur à l'École nationale supérieure d'architecture de Nantes, chercheur au Centre de recherche nantais architectures urbanités (Crenau), laboratoire « Ambiances, architectures, urbanités » (AAU, UMR CNRS 1563)

laurent.lescop@nantes.archi.fr

\section{JEAN-MICHEL GENESTE}

Conservateur général du patrimoine honoraire, équipe de recherche de recherche de la grotte Chauvet-Pont d'Arc ; laboratoire Pacea (UMR CNRS 5199 / université de Bordeaux / ministère de la Culture) 
jeanmichelgeneste@orange.fr

\section{MARTIN MARQUET}

Producteur, Rup'Art Productions

martin.marquet@me.com

VICTOR YVIN

Architecte

victor.yvin2@gmail.com

PACÔME GÉRARD

Architecte

pacome.gerard@gmail.com

PASCAL MAGONTIER

Auteur, réalisateur

magontier.p@orange.fr 